\title{
BMJ Does smoking cessation with varenicline Open worsen vascular endothelial function?
}

\author{
Akira Umeda, ${ }^{1}$ Toru Kato, ${ }^{2}$ Tateki Yamane, ${ }^{1}$ Heiichi Yano, ${ }^{1}$ Tamio leiri, ${ }^{1}$ \\ Kazuya Miyagawa, ${ }^{3}$ Hiroshi Takeda, ${ }^{3}$ Yasumasa Okada ${ }^{4}$
}

To cite: Umeda A, Kato T, Yamane T, et al. Does smoking cessation with varenicline worsen vascular endothelial function?. BMJ Open 2013;3:e003052. doi:10.1136/bmjopen-2013003052

- Prepublication history for this paper is available online. To view these files please visit the journal online (http://dx.doi.org/10.1136/ bmjopen-2013-003052).

Received 14 April 2013 Revised 18 May 2013 Accepted 30 May 2013

This final article is available for use under the terms of the Creative Commons Attribution Non-Commercial 3.0 Licence; see http://bmjopen.bmj.com

\footnotetext{
${ }^{1}$ Department of Internal Medicine, International University of Health and Welfare Shioya Hospital, Tochigi, Japan

2Department of Clinical Research, National Hospital Organization Tochigi Medical Center, Tochigi, Japan

${ }^{3}$ Department of Pharmacology, School of Pharmacy, International University of Health and Welfare, Tochigi, Japan ${ }^{4}$ Department of Internal Medicine, National Hospital Organization Murayama Medical Center, Tokyo, Japan
}

Correspondence to Dr Akira Umeda; aumeda@hf.catv.ne.jp

\section{ABSTRACT}

Objectives: A meta-analysis suggested that the use of varenicline, which is a partial agonist of nicotinic acetylcholine receptors and is effective in smoking cessation, increases the risk of cardiovascular events within 52 weeks of starting treatment. Defining these events as occurring during drug treatment (usually for 12 weeks) or within 30 days of discontinuation, another meta-analysis showed that the risk was statistically insignificant. In the present study, we aimed to clarify the effect of varenicline-assisted smoking cessation on vascular endothelial function assessed by flow-mediated vasodilation (FMD).

Design: Before-after and time-series.

Setting: Tochigi Prefecture, Japan.

Participants: Data of 85 participants who visited nicotine-dependent outpatient services were reviewed. FMD was repeatedly measured in 33 of the 85 participants. Inclusion criteria: 20 years and older, Brinkman index $\geq 200$, Tobacco Dependence Screener $\geq 5$ and stated motivation to quit smoking.

Interventions: Each participant was treated with varenicline titrated up to $1.0 \mathrm{mg}$ twice daily (for 12 weeks in total).

\section{Primary and secondary outcome measures:}

Participants were evaluated by FMD prior to, and 3 months after, complete smoking cessation. Follow-up FMD measurements were carried out every 3 months if possible. Changes in FMD during varenicline use were also evaluated.

Results: FMD was significantly increased from $4.0 \pm 1.8 \%$ to $5.5 \pm 2.2 \%(p<0.01, n=22) 3$ months after complete cessation. Although the timecourse of FMD in most of the cases showed an increase with fluctuations, there was an exceptional case where FMD decreased over the 9 months following complete cessation. Although statistically insignificant, FMD also increased during varenicline use (from $3.7 \pm 2.7 \%$ to $4.3 \pm 2.8 \%, n=11$ ).

Conclusions: Our observations suggest that in ceasing smokers, varenicline and smoking cessation do not lead to a worsening of the vascular endothelial function.

Trial registration: FK-79 (International University of Health and Welfare).

\section{INTRODUCTION}

Smoking disturbs endothelial function, ${ }^{1-3}$ and smoking cessation reduces the risk of

\section{ARTICLE SUMMARY}

Article focus

- Although varenicline is effective in smoking cessation, the risk of adverse cardiovascular events was raised in some reports, although the statistical significance of this risk was inconsistent across the reports.

\section{Key messages}

- This paper demonstrated that vascular endothelial function improved after successful smoking cessation with varenicline and adds to the evidence that the risk of adverse cardiovascular events due to varenicline is minimal.

Strengths and limitations of this study

- Participants who relapsed were not fully followed up with FMD. They usually stopped visiting. Another key control group which stopped smoking without varenicline is missing. Further prospective studies including these control groups are necessary to clarify whether the use of varenicline in smoking cessation worsens or improves vascular endothelial function.

thromboembolic events. ${ }^{4}$ Various pharmacotherapies have been successfully applied to assist smoking cessation. ${ }^{5}$ Smoking cessation rates with varenicline, which is a partial agonist of the $\alpha 4 \beta 2$ nicotinic acetylcholine receptor, have been reported to be better than previous drugs such as bupropion and the nicotine patch. ${ }^{6}{ }^{7}$ Varenicline has also been reported to be safe, well tolerated and satisfactory to patients. ${ }^{8}$ Therefore, varenicline is currently the most effective and frequently used drug for the purpose of quitting smoking in Japan.

In 2010, Rigotti et al reported that the incidence of cardiovascular events was statistically insignificant (varenicline group $7.1 \%$ vs placebo group 5.7\%; difference, $1.4 \%$; $95 \%$ CI -2.3 to 5.0) in a Pfizer-sponsored study of 714 smokers with stable cardiovascular disease. Nevertheless, this increased risk with varenicline led the US Food and Drug Administration to call for a systematic review of 
all randomised clinical trials of varenicline for tobacco cessation to determine its association with cardiovascular risk. ${ }^{10}{ }^{11}$ A meta-analysis by Singh $e t$ al $^{12}$ in 2011 suggested that the use of varenicline for smoking cessation increases the risk of cardiovascular disease. However, the conclusion from another meta-analysis by Prochaska and Hilton ${ }^{13}$ in 2012 was that the risk of cardiovascular events associated with varenicline use was statistically insignificant. Two reasons for the conflicting findings were described by Prochaska et al: (1) Prochaska et al defined the varenicline-related cardiovascular events as occurring during treatment (usually for 12 weeks) or within 30 days of discontinuation, while the analysis by Singh et al contained data for 52 weeks. (2) The Peto ORs which Singh et al used are far more extreme than the Mendel-Haenszel ORs which well match the relative risks. These reports lead us to hypothesise that varenicline-assisted smoking cessation worsens vascular endothelial function, which is an important determinant in maintaining smooth blood flow and prevention of atherosclerosis. ${ }^{14} 15$

We tried to obtain details on the timing of serious cardiovascular adverse events by contacting the medical adviser for varenicline at Pfizer. It was indicated that the incidence of cardiovascular events during the use of varenicline was low and statistically insignificant until the phase 3 trial $(n=3267)$. The precise contents of cardiovascular events are: angina pectoris one case $(<0.1 \%)$, palpitation 15 cases $(0.4 \%)$ and tachycardia five cases $(0.1 \%)$. Therefore, the official drug information documents did not describe a contraindication for patients with ischaemic heart disease or other active heart diseases. After the start of use in Japan in May 2008, nine serious cardiovascular events during use of varenicline (or 1-2 days after the discontinuation) were reported from the company to the Japanese Ministry of Health, Labor and Welfare as postmarketing information. The risk of cardiovascular events associated with varenicline use was thought to be statistically insignificant, and therefore no special alert was announced by the government. Data of cardiovascular events after the discontinuation of varenicline were not available from the company.

In the present study, we aimed to clarify whether smoking cessation with varenicline worsens or improves vascular endothelial function by reviewing the data of flow-mediated vasodilation (FMD), which is a noninvasive method to evaluate the vascular endothelial function and is a surrogate endpoint of vascular health, reflecting endothelial nitric oxide release. ${ }^{16-19}$

\section{METHODS}

\section{Enrolment}

Inclusion criteria were 20 years and older, Brinkman index (equivalent to the $20 \times$ pack years) $\geq 200$, a nicotine dependence score (Tobacco Dependence Screener, $\mathrm{TDS})^{20} \geq 5$, and a stated motivation to quit smoking. These criteria were established by the Japanese-drug use system for nicotine-dependent outpatients. Therefore, this report is a retrospective study in which we evaluated the data of normal nicotine-dependent outpatient services at the International University of Health and Welfare Shioya Hospital.

\section{Treatment with varenicline}

Each participant was treated with varenicline titrated up to $1.0 \mathrm{mg}$ twice daily $(0.5 \mathrm{mg}$ once daily for 3 days, then $0.5 \mathrm{mg}$ twice daily for 4 days, then $1.0 \mathrm{mg}$ twice daily for 11 weeks). The target quit date was planned to be 7 days after the initiation of varenicline (day 8). Participants were encouraged to measure body weight frequently and not to eat excessively. After the first visit (day 1), visit dates were planned on days 15, 29, 57, 85 and 99 . Self-reported smoking status and exhaled-carbon monoxide concentration were assessed at each visit. Vital signs, body weight and adverse event information were collected at each visit. Blood was sampled at baseline (day 1) and at 3 months (usually on day 99) for biochemical analysis. Varenicline was discontinued on day 85 .

\section{Study procedures}

FMD data were examined before smoking cessation and at 3 months after complete cessation (usually on day 99). If a participant succeeded in complete smoking cessation (zero cigarettes/day) on day 8, 3 months of no smoking would occur on day 99. Participants were encouraged to visit every 3 months for FMD follow-up measurements for 1 year. Smoking status was assessed at every visit by self-report and exhaled-carbon monoxide concentration. Additionally, we examined the FMD data during varenicline use. We compared the FMD data before varenicline use (day 1) and under varenicline use (between days 29 and 85). In the beginning of this study, we thought that FMD change (before and after smoking cessation) should be evaluated with the same other conditions. As the baseline FMD was measured without varenicline (day 1, before taking the first tablet), the second measure was carried out when varenicline was thought to be washed out from the body (day 99). After observing statistical significance, we started the comparison between the baseline (day 1) and under varenicline use (between days 29 and 85).

\section{Measurement of FMD using an ultrasound system}

Endothelial function was evaluated by measuring the FMD of the brachial artery (BA) in a core ultrasound laboratory using a standardised protocol according to the international guidelines and Japanese guidelines from the Vascular Failure Working Group. ${ }^{19} 21$ We used an ultrasound system equipped with an edge-tracking system for two-dimensional imaging and a pulsed Doppler flow velocimeter for automatic measure ment (UNEXEF38G; Unex Co. Ltd, Nagoya, Japan). Regarding the reproducibility of the FMD measurements using this system, the correlation coefficient between the two examinations was reported to be 0.86 with a coefficient of variance of $11.2 \% .^{22}$ In brief, the diameter of 
the $\mathrm{BA}$ at rest was measured in the cubital region, and subsequently the cuff was inflated to $50 \mathrm{~mm} \mathrm{Hg}$ above systolic blood pressure for $5 \mathrm{~min}$ and then deflated. The diameter at the same point was monitored continuously, and the maximum dilation of the BA was confirmed and measured by a plateau or no increase of the diameter using real-time monitoring for at least $1 \mathrm{~min}$ after cuff deflation. FMD was calculated as follows: FMD $(\%)=$ (maximum diameter - diameter at rest $) \times 100 /$ diameter at rest.

\section{Data analysis}

Data are shown as mean \pm SD. Student's unpaired t test was used for the comparison between participants who relapsed and abstained (two-tailed). Spearman's regression analysis was used for the evaluation of the relationship between baseline FMD and BA diameter. Student's paired t test was used for the comparison between baseline and after the smoking cessation (two-tailed). Statistical significance was set at $\mathrm{p}<0.05$.

\section{RESULTS}

\section{Participant characteristics}

The demographics of the smokers $(n=74)$ who visited the smoking cessation outpatient service at the International University of Health and Welfare Shioya Hospital from June 2010 to October 2012 are shown in table 1. Age, Brinkman index, current smoking status, exhaled-carbon monoxide concentration and TDS at the first visit did not show any significant differences between participants who relapsed or abstained. The status of participants who relapsed or abstained was determined 3 months after the original abstinence. Among those who abstained, 22 were evaluated for changes in FMD between the baseline and after the discontinuation of varenicline.

\section{Evaluation of change in FMD before and after smoking cessation with varenicline}

Among participants who quit smoking, FMD increased from $4.0 \pm 1.8 \%$ to $5.5 \pm 2.2 \% \quad(\mathrm{p}=0.00081, \mathrm{n}=22) \quad$ in
3 months after complete smoking cessation (figure 1). There was no significant correlation between baseline FMD and BA diameter. In 6 of 22 cases, FMD had decreased at 3 months as compared with the baseline. We could observe the FMD time-course for periods longer than 9 months after smoking cessation in 10 cases (figure 2). In cases 1, 2, 6, 7, 9 and 10, FMD appeared to increase while fluctuating. In case 4, FMD appeared not to change. In case 5, FMD appeared to be the best at 6 months after smoking cessation. In case 8 , FMD appeared to be the best at 3 months after smoking cessation. In case 3, FMD appeared to 'decrease' while fluctuating.

\section{Change of data associated with atherosclerosis in FMD-evaluated participants}

Body weight and body mass index increased significantly at 3 months after complete smoking cessation $(n=22$, table 2). None of the other data (systolic or diastolic blood pressure, low-density lipoprotein cholesterol, triglycerides, glycated haemoglobin and blood sugar) showed any significant differences between baseline and at 3 months after complete smoking cessation with varenicline.

\section{Evaluation of change in FMD before and during the use of varenicline}

We could observe the FMD change before and during the use of varenicline in 11 cases since November 2012. Although statistically insignificant, FMD also increased from $3.7 \pm 2.7 \%$ (before varenicline use: day 1 ) to 4.3 $\pm 2.8 \%$ (during varenicline use: between days 29 and 85 ).

\section{DISCUSSION}

Endothelial cells form a single layer that covers the inside of blood vessels, and the endothelial function is an important determinant in maintaining smooth blood flow and prevention of atherosclerosis. ${ }^{14-17}$ Johnson et $a l^{23}$ reported that FMD increased after smoking cessation with five pharmacotherapies (nicotine lozenge, nicotine patch, sustained-release bupropin, nicotine patch plus nicotine lozenge and sustained-release

Table 1 Demographics of smokers who visited the smoking cessation outpatient service at the International University of Health and Welfare Shioya Hospital from June 2010 to October 2012

\begin{tabular}{lllll}
\hline & All participants & Relapsed & $\begin{array}{l}\text { Abstained } \\
\text { (FMD-evaluated) }\end{array}$ & $\begin{array}{c}\text { p Values relapsed } \\
\text { vs abstainer }\end{array}$ \\
\hline $\mathrm{N}$ & 74 & 26 & $48(22)$ & $41 / 7(19 / 3)$ \\
Male/female & $61 / 13$ & $20 / 6$ & $53.0 \pm 14.9(53.0 \pm 15.0)$ & 0.904 \\
Age & $53.2 \pm 13.6$ & $53.5 \pm 11.0$ & $817 \pm 557(870 \pm 600)$ & 0.988 \\
Brinkman index & $817 \pm 525$ & $819 \pm 466$ & $25.3 \pm 10.1(26.0 \pm 11.5)$ & 0.780 \\
Current smoking (cigarettes/day) & $25.5 \pm 10.1$ & $26.0 \pm 10.2$ & $29.0 \pm 12.6(29.1 \pm 13.5)$ & 0.175 \\
Exhaled carbon monoxide (ppm) & $30.9 \pm 15.3$ & $34.4 \pm 19.5$ & $8.1 \pm 2.9(8.7 \pm 3.7)$ & 0.998 \\
Nicotine dependence score & $8.1 \pm 1.5$ & $8.1 \pm 1.2$ & & \\
(Tobacco Dependence Screener) & & & & \\
\hline $\begin{array}{l}\text { Data (age, sex, Brinkman index, cigarettes/day, expired carbon monoxide and nicotine dependence score) were obtained on the first-visit day } \\
\text { (mean } \pm \text { SD). The FMD-evaluated participants referenced in this article are included in the abstained column. }\end{array}$
\end{tabular}




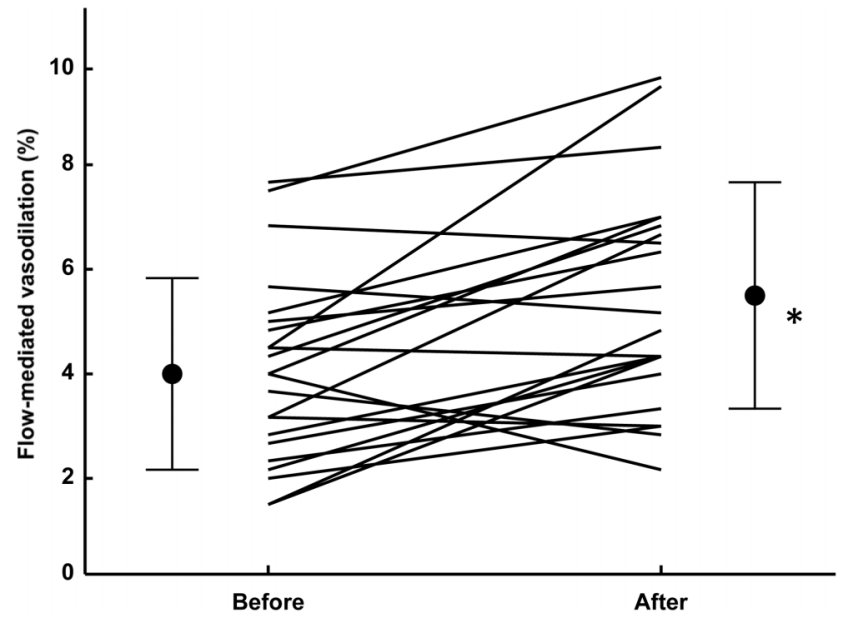

Figure 1 Change of flow-mediated vasodilation (FMD) between prior to and 3 months after complete smoking cessation with varenicline. FMD was significantly increased after smoking cessation $\left({ }^{*} p<0.01\right.$ compared with baseline, $\mathrm{n}=22$, error bars: SD). Varenicline was discontinued 2 weeks before the second FMD measurement. In six cases, FMD decreased slightly, but in total FMD increased significantly (improved) at 3 months after complete smoking cessation $(p=0.00081)$.

bupropion plus nicotine lozenge). The reported increase in FMD in those who quit smoking was $1 \%$ $(6.2-7.2 \%)$ at 1 year after the smoking cessation date. They also reported that FMD did not change in those who continued to smoke. In the present study, we aimed to address this issue by examining changes in FMD following smoking cessation with varenicline. Individuals who stopped smoking experienced a significant improvement in endothelial function, despite gaining weight. An increase in body weight with smoking cessation is consistent with the previous findings. ${ }^{24}$ The improvement at 3 months after smoking cessation shown in our FMD data was about the same degree as Johnson et $a l^{23}$ found at 1 year. The $1.5 \%$ absolute increase in FMD among smokers is not as dramatic as that reported with statins and other interventions. ${ }^{19}$

Johnson et $a l^{23}$ found that BA diameter, reactive hyperaemia flow, exhaled-carbon monoxide concentration and high-density lipoprotein cholesterol were independently associated with baseline FMD through multivariate regression analysis. They reported that the correlation was the strongest between BA diameter and FMD. Other significantly correlated factors were reported to be age, systolic and diastolic blood pressures, cigarettes smoked per day, current pack-years, creatine, glucose and highsensitivity $\mathrm{C}$ reactive protein. They also showed an improvement in FMD on the occasion of smoking cessation after controlling for changes in BA diameter and other factors of reactive hyperaemia, low-density lipoprotein cholesterol and home smoking bans. On the other hand, there was no significant correlation between BA diameter and baseline FMD in our data. We also conducted multivariate regression analysis, but no factors listed above were found to be associated with baseline FMD. One reason for this negative association may be that the number of smokers who participated in our study was much smaller than that reported by Johnson et al $(\mathrm{n}=1504)$. Also, while their data were from Caucasian (83.9\%) and African-American (13.6\%) participants, our data were $100 \%$ from Asians. Johnson et al

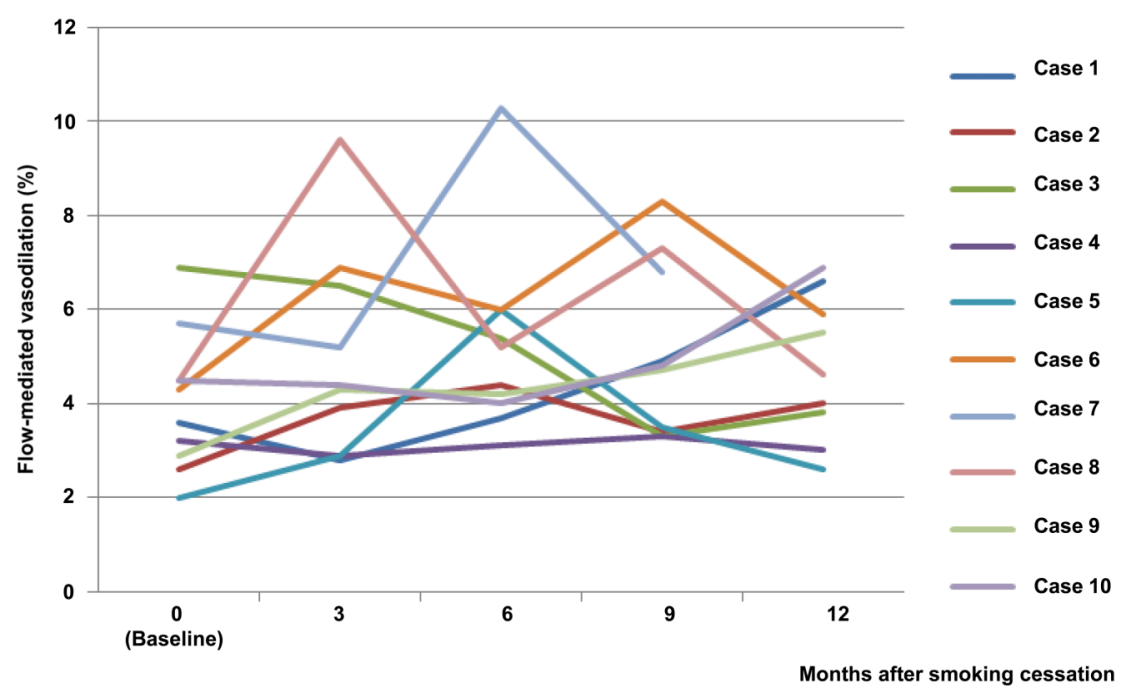

Figure 2 Time-course of flow-mediated vasodilation (FMD) data before and after smoking cessation. All participants succeeded in smoking cessation during this evaluation. An observation period greater than 9 months was possible in 10 cases. Cases 1 , 2 , 6, 7, 9 and 10: FMD appeared to increase, although fluctuating. Case 4: FMD appeared not to change. Case 5: FMD increased after smoking cessation and appeared to peak at 6 months, then decrease. Case 8: FMD appeared to be the best at 3 months after smoking cessation. Case 3: FMD appeared to 'decrease' while fluctuating. No cardiovascular events were found in these participants until now. 
Table 2 Changes in the data which are related to atherosclerosis besides smoking: comparison between baseline and at 3 months after complete smoking cessation with varenicline

\begin{tabular}{lccc}
\hline & Abstained baseline & Abstained 3 months & $\begin{array}{l}\text { p Value abstained baseline } \\
\text { vs } \mathbf{~ m o n t h s ~}\end{array}$ \\
\hline Body weight $(\mathrm{kg})$ & $64.9 \pm 12.0$ & $66.3 \pm 12.1$ & $0.001^{*}$ \\
Body mass index $\left(\mathrm{kg} / \mathrm{m}^{2}\right)$ & $23.4 \pm 3.3$ & $23.9 \pm 3.4$ & $0.001^{*}$ \\
Systolic blood pressure $(\mathrm{mm} \mathrm{Hg})$ & $125.5 \pm 14.3$ & $123.0 \pm 11.2$ & 0.382 \\
Diastolic blood pressure $(\mathrm{mm} \mathrm{Hg})$ & $73.2 \pm 9.0$ & $72.9 \pm 9.0$ & 0.880 \\
Low-density lipoprotein cholesterol $(\mathrm{mg} / \mathrm{dL})$ & $116.3 \pm 27.0$ & $116.4 \pm 21.9$ & 0.985 \\
Triglyceride $(\mathrm{mg} / \mathrm{dL})$ & $166.0 \pm 76.0$ & $231.7 \pm 355.3$ & 0.331 \\
Glycated aaemoglobin (\%) & $5.57 \pm 0.65$ & $5.65 \pm 1.08$ & 0.540 \\
Blood sugar $(\mathrm{mg} / \mathrm{dL})$ & $111.0 \pm 28.7$ & $108.2 \pm 25.0$ & 0.551 \\
\hline Blood was taken at around 13:00-17:00. Data are from participants who were evaluated with FMD (N=22; mean $\left.\pm \mathrm{SD} ;{ }^{*}<0.05\right)$.
\end{tabular}

reported FMD data at baseline and after 1 year only. Therefore, the present study is the first report on the timecourse of FMD following smoking cessation.

It must be noted that Harrison-Woolrych $e t a l^{25}$ reported that patients who were prescribed varenicline could be associated with an increased risk of cardiovascular events that they attributed partly to coronary artery spasms induced by varenicline. Nevertheless, our observation data on FMD change before and during the use of varenicline suggested that this drug is not harmful to endothelial function. Although statistically insignificant, FMD even increased a little $(0.6 \%)$ during varenicline use.

A key control group which took varenicline but failed to stop smoking was not carefully followed up with FMD. Participants who relapsed usually stopped visiting. We could observe only one time course of FMD in a participant who used varenicline but could not achieve smoking cessation. The time course showed a decrease in FMD with fluctuations (figure 3). Another key control group which stopped smoking without varenicline is missing. Further prospective studies including these control

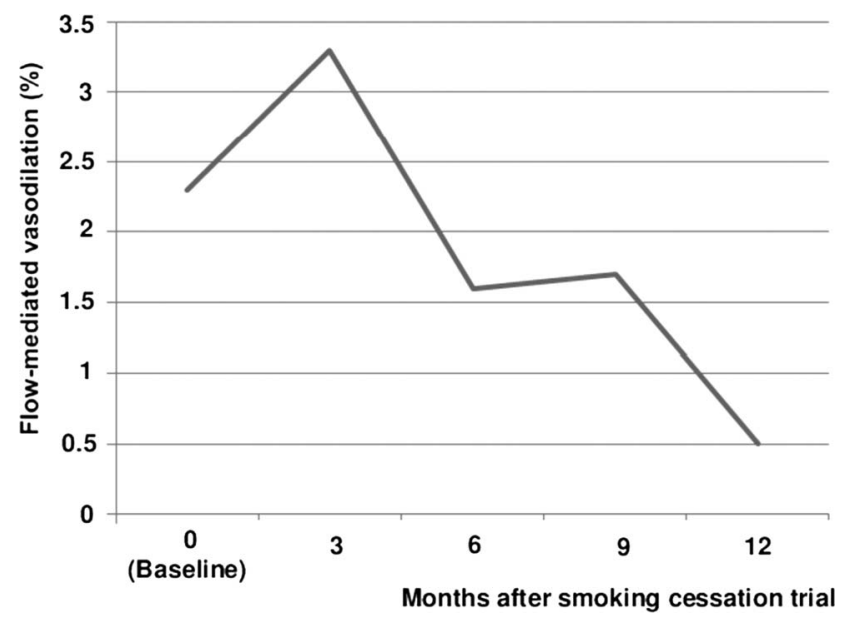

Figure 3 Timecourse of flow-mediated vasodilation (FMD) data before and after a smoking cessation trial in a relapsed participant. This participant could not achieve smoking cessation, but FMD follow-up was possible. FMD appeared to 'decrease' while fluctuating. groups are necessary to clarify completely whether the use of varenicline in smoking cessation worsens or improves vascular endothelial function.

In conclusion, our observations suggest that in ceasing smokers, varenicline and smoking cessation do not lead to a worsening of vascular endothelial function, which could be used in antismoking campaigns or by smoking cessation services.

Contributors AU and TK conceived the idea of the study. AU was responsible for undertaking the data analysis and produced the tables and graphs.

The initial draft of the manuscript was prepared by AU, TK and $Y 0$ and then circulated repeatedly among all authors for critical revision. AU was responsible for the acquisition of the data and TK, TY, HY, TI, KM, HT and YO contributed to the interpretation of the results. All authors read and approved the final manuscript.

Funding This work was supported by the International University of Health and Welfare.

\section{Competing interests None.}

Ethics approval The institute's ethical committee approved the access to patient records and confirmed that patient confidentiality was maintained.

Provenance and peer review Not commissioned; externally peer reviewed. Data sharing statement No additional data are available.

\section{REFERENCES}

1. Celermajer DS, Sorensen KE, Georgakopoulos D, et al. Cigarette smoking is associated with dose-related and potentially reversible impairment of endothelium-dependent dilation in healthy young adults. Circulation 1993;88:2149-55.

2. Celermajer DS, Adams MR, Clarkson $P$, et al. Passive smoking and impaired endothelium-dependent arterial dilatation in healthy young adults. N Engl J Med 1996;334:150-4.

3. Kato T, Inoue T, Morooka T, et al. Short-term passive smoking causes endothelial dysfunction via oxidative stress in nonsmokers. Can J Physiol Pharmacol 2006;84:523-9.

4. Rosenberg L, Kaufman DW, Helmrich SP, et al. The risk of myocardial infarction after quitting smoking in men under 55 years of age. N Engl J Med 1985;313:1511-4.

5. Bader P, McDonald P, Selby P. An algorithm for tailoring pharmacotherapy for smoking cessation: results from a Delphi pane of international experts. Tob Control 2009;18:34-42.

6. Zhu S-H, Lee M, Zhuang Y-L, et al. Interventions to increase smoking cessation at the population level: how much progress has been made in the last two decades? Tob Control 2012;21:110-8.

7. Keating GM, Lyseng-Williamson KA. Varenicline: a pharmacoeconomic review of its use as an aid to smoking cessation. Pharmacoeconomics 2010;28:231-54 
8. Etter JF, Schneider NG. An internet survey of use, opinions and preferences for smoking cessation medications: nicotine, varenicline, and bupropion. Nicotine Tob Res 2013;15:59-68.

9. Rigotti NA, Pipe AL, Benowitz NL, et al. Efficacy and safety of varenicline for smoking cessation in patients with cardiovascular disease: a randomized trial. Circulation 2010;121:221-9.

10. FDA. Chantix (varenicline): Label Change-Risk of Certain Cardiovascular Adverse Events. [online, updated 07/22/2011]. http:// www.fda.gov/Safety/MedWatch/Safetylnformation/ SafetyAlertsforHumanMedicalProducts/ucm259469.htm

11. FDA. FDA Drug Safety Communication: Chantix (varenicline) may increase the risk of certain cardiovascular adverse events in patients with cardiovascular disease. [online, 06/16/2011]. http://www.fda. gov/Drugs/Drugsafety/ucm259161.htm

12. Singh S, Loke YK, Spangler JG, et al. Risk of serious adverse cardiovascular events associated with varenicline: a systematic review and meta-analysis. CMAJ 2011;183:1359-66.

13. Prochaska JJ, Hilton JF. Risk of cardiovascular serious adverse events associated with varenicline use for tobacco cessation: systematic review and meta-analysis. BMJ 2012;344:e2856.

14. Vanhoutte PM. Endothelium and control of vascular function. Hypertension 1989;13:658-67.

15. van Hinsbergh VW. Endothelium-role in regulation of coagulation and inflammation. Semin Immunopathol 2012;34:93-106.

16. Celermajer DS, Sorensen KE, Gooch VM, et al. Non-invasive detection of endothelial dysfunction in children and adults at risk of atherosclerosis. Lancet 1992;340:1111-5.

17. Davignon J, Ganz P. Role of endothelial dysfunction in atherosclerosis. Circulation 2004;109:III-27-32.
18. Santos-García D, Blanco M, Serena J, et al. Brachial arterial flow mediated dilation in acute ischemic stroke. Eur $J$ Neurol 2009;16:684-90.

19. Corretti MC, Anderson TJ, Benjamin EJ, et al. Guidelines for the ultrasound assessment of endothelial-dependent flow-mediated vasodilation of the brachial artery: a report of the International Brachial Arterial Reactivity Task Force. J Am Coll Cardiol 2002;39:257-65.

20. Kawakami N, Takatsuka N, Inaba S, et al. Development of a screening questionnaire for tobacco/nicotine dependence according to ICD-10, DSM-III-R, and DSM-IV. Addict Behav 1999;24:155-66.

21. Inoue $\mathrm{T}$, Matsuoka $\mathrm{H}$, Higashi $\mathrm{Y}$, et al. Flow-mediated vasodilation as a diagnostic modality for vascular failure. Hypertens Res 2008;31:2105-13.

22. Tomiyama $\mathrm{H}$, Matsumoto $\mathrm{C}$, Yamada J, et al. The relationships of cardiovascular disease risk factors to flow-mediated dilatation in Japanese subjects free of cardiovascular disease. Hypertens Res 2008;31:2019-25.

23. Johnson HM, Gossett LK, Piper ME, et al. Effects of smoking and smoking cessation on endothelial function: 1-year outcomes from a randomized clinical trial. J Am Coll Cardiol 2010;55:1988-95.

24. Aubin HJ, Farley A, Lycett D, et al. Weight gain in smokers after quitting cigarettes: meta-analysis. BMJ 2012;345:e4439.

25. Harrison-Woolrych M, Maggo S, Tan M, et al. Cardiovascular events in patients taking varenicline: a case series from intensive postmarketing surveillance in New Zealand. Drug Saf 2012;35:33-43. 